\title{
(C) OPEN ACCESS \\ Sleep deprivation and starvation in hospitalised patients: how medical care can harm patients
}

\author{
Tim Xu, ${ }^{1}$ Elizabeth C Wick, ${ }^{1,2}$ Martin A Makary ${ }^{1,2}$
}

${ }^{1}$ Johns Hopkins School of Medicine, Baltimore, Maryland, USA

${ }^{2}$ Department of Surgery, Johns Hopkins Hospital, Baltimore, Maryland, USA

\section{Correspondence to} Dr Martin Makary, Johns Hopkins School of Medicine, Baltimore, MD 21224, USA; mmakary1@jhmi.edu

Received 14 May 2015 Revised 13 August 2015 Accepted 17 August 2015 Published Online First 8 September 2015
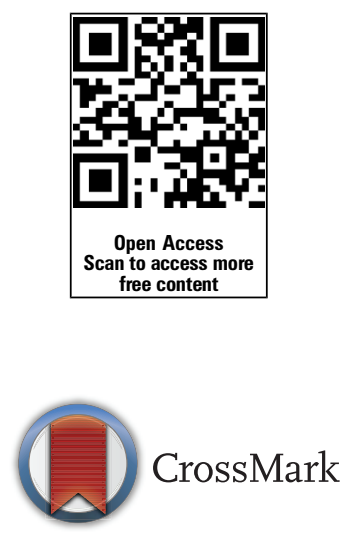

To cite: $X u T$, Wick EC, Makary MA. BMJ Qual Saf 2016:25:311-314.
The field of patient safety has focused on acute adverse events. However, hospitalised patients face regular stressors, such as sleep deprivation and malnutrition, which frequently predispose them to other complications, yet are unlikely to be detected as preventable adverse events in chartbased studies. Unlike easy-to-measure patient safety events, such as retained foreign objects or catheter infections, sleep deprivation and malnutrition are more difficult to measure, as they are dynamic and occur with varying severity. Even a young, extremely healthy person at home without illness will become physiologically stressed and transiently immunocompromised after a mere $24 \mathrm{~h}$ of starvation and a poor night's sleep. ${ }^{1}$ These stressors alone can result in acne, fatigue, oral ulcers and impaired judgment-conditions that in the presence of illness can worsen or obscure the clinical picture being evaluated by clinicians. In frail patients, the stressors of sleep deprivation and malnutrition are magnified further, and, in conjunction with the stress of the patient's underlying medical condition, can overwhelm a patient's physiological reserve and lead to or worsen complications. ${ }^{2}$

Consider a 65-year-old woman who presents to the emergency department (ED) for pneumonia. She has not eaten or had anything to drink for a few days due to not feeling well. Because the doctors are unsure if the admitting doctor may want to perform a procedure, she is placed on nil per os (NPO) status until the admitting team can come to evaluate her. The patient waits in a noisy ED with devices beeping and people talking loudly outside her room, shared with another patient-a room she will stay in for an additional full day since the hospital ward is full. Including the time in the ED waiting room, and waiting for an admitting service to arrive, the patient spends nearly $12 \mathrm{~h}$ until she is seen by the trainee rotating on the pulmonary service, who keeps the patient NPO until the senior staff physician is available to hear the patient's full story. Now, on hospital day \#2, the consultant decides to perform a bronchoscopy, but, because it is unclear whether the endoscopy suite will have the support staff to accommodate the procedure the next day, the patient is put on a wait list and stays NPO in the meantime. The following day, it is performed as the last case of the day. The next afternoon the patient is fed for the first time in 6 days with soup and Jell-O, including the 3 days the patient did not eat before presenting to the hospital. Ultimately, her diet is slowly advanced, and the patient is discharged a few days later. Four days later, the patient is readmitted complaining of the same symptoms for which she initially presented. She is managed expectantly and discharged again 2 days later in a weak and frail state. She is asked to complete a patient satisfaction survey and responds with a low-satisfaction rating of her experience.

\section{COULD WE HAVE DONE BETTER?}

In our personal experience as surgeons at a busy tertiary hospital, the goal of avoiding unnecessary malnutrition and sleep deprivation in our patients has all too often been forgotten or prioritised far behind other more technical goals of surgical care. But keeping a patient strong and rested is a critical goal towards the same end. Streamlining good medical care requires a systems approach and a change in safety culture. In this Viewpoint, we explore ways to improve quality by adopting strategies to prevent unnecessary sleep deprivation and malnutrition that are commonplace in inpatient care. 
The problem of sleep deprivation and malnutrition stressing patients is endemic yet further compounded by the complexity and changes in the way medical care is delivered today. In some countries, the problem is worsened when hospitals are operating close to full capacity, a trend we have observed at our own hospital and many others. As a result, more patients are spending the night in noisy emergency rooms, recovery rooms and even hallways that lack privacy. Over the last decade, emergency room wait times in the USA and other countries have increased, and some hospitals have observed the growing trend of patients spending the night in the recovery room after elective surgery. This may be due to inefficiencies in admitting patients from the emergency room and bed flow bottlenecking. Longer wait lists for procedures (eg, radiology interventions, endoscopy, etc) require more last-minute rescheduling, stretching the time patients are without nutrition (NPO). Financial pressures affecting hospitals have already impacted staffing levels, patient unit assignments and bed flow. Finally, a large number of specialty services and associated trainees means communication layers are complex, resulting in communication breakdowns scheduling inpatient tests and procedures. All of these disruptions in patients' physiology may contribute to what Detsky and Krumholz describe as 'posthospital syndrome' and manifest as rehospitalisations. ${ }^{3}$

\section{Rethinking nutrition}

Malnutrition affects as many as a third to a half of all hospitalised patients and can lead to chronic inflammatory processes, muscle breakdown and organ damage. ${ }^{4}$ Many patients are malnourished to begin with due to their illness and multiple comorbidities, which can be compounded by wound healing and disruptions in food intake following surgery. A study of US national administrative claims data showed that only $3 \%$ of adult hospital admissions include a diagnosis of malnutrition, suggesting that it remains under-recognised. ${ }^{4}$ Few patients receive nutritional consults despite evidence that malnutrition leads to longer length of stay, mortality and increased risk of other adverse events, such as falls. ${ }^{5}$ Another barrier is the notoriously poor-quality food served at hospitals, representing a highly processed food offering from inexpensive food suppliers. Hospitals have an opportunity to promote healthy foods and improve nutrition by serving better food. Some have taken the lead by serving local whole foods that are organic and achieve a broad range of nutritional goals.

In some cases, malnutrition can be stemmed by adopting standardised preoperative protocols. The American Society of Anesthesiologists recommends avoiding extended preoperative fasting and endorses clear liquids up to $2 \mathrm{~h}$ before surgery, but adoption of this practice has been slow. ${ }^{6}$ Limiting preoperative fasting is an essential component of Enhanced
Recovery after Surgery (ERAS) protocols, which aim to restore physiological function and facilitate recovery. The evidence shows that needless prolongation of fasting after gastrointestinal surgery actually increases the risk of infection and does not reduce the risk of complications such as anastomotic dehiscence. ${ }^{7}$ Other components of ERAS are summarised in the box 1. At our institution, implementing the comprehensive ERAS protocol has led to a significant reduction in postoperative complications, hospital length of stay and improved patient satisfaction. ${ }^{8}$

These principles of nutrition in patients with gastrointestinal surgery, who have some of the strictest dietary requirements, can be applied to other patients. Patients can benefit from a carbohydrate-rich drink $2 \mathrm{~h}$ prior to surgery unless the patient has a gastrointestinal obstruction, dysmotility or another special circumstance. After surgery, patients can restart an oral diet at will and be evaluated for nutritional supplements. For example, specific metabolic modulators like arginine and fish oils have been found to reduce the risk of surgical site infections and length of stay. ${ }^{5}$ Second, any potential interruption to restoring the patient's ability to eat should be seriously considered. For example, multimodal pain regimens should always be considered to avoid excessive opioid use, which can delay return of normal bowel function and cause postoperative ileus. ${ }^{7}$ Physicians should also create prescreening tools for high-risk patients and build a culture of awareness that empowers the entire care team to prevent malnutrition, from pre surgery to post discharge. Above all, physicians, nurses and other staff should all be aware that extended periods without food stresses a patient's physiological reserves and staff can be educated to question a patient's NPO status frequently.

\section{Promoting rest}

The hospital environment can be noisy with alarms, phones, hallway conversations and patients sharing a

\section{Box 1 Common enhanced recovery protocol elements}

Patient education and engagement strategies Nutritional optimisation before surgery

- Carbohydrate drink a few hours before surgery

- Pre-emptive treatment of postoperative nausea and vomiting

- Multimodal pain management with avoidance of narcotics

- Goal directed intravenous fluids in the operating roomEarly mobility post surgery

- Avoidance of post-operative fasting with early resumption of oral intake

- Avoidance of tubes and drains

Avoidance of intravenous fluids after surgery 
room. These are then compounded by intercoms, chatter, lights, plus sometimes frequent interruptions at night for medications, vital signs and lab draws. WHO recommends that hospitals keep noise levels below $35 \mathrm{~dB}$ at night and $40 \mathrm{~dB}$ during the day. Recent studies suggest, however, that many hospitals far exceed these guidelines, with one study finding noise levels greater than $55 \mathrm{~dB} 70-90 \%$ of the time and spikes as high as $85 \mathrm{~dB}$, which is the equivalent of busy city traffic. ${ }^{9}$ In a prospective cohort study of patients with myocardial infarction, researchers replaced ceiling tiles with sound-absorbing surfaces and found a significant reduction in sympathetic arousal and intravenous $\beta$-blocker use. ${ }^{10}$ Interestingly, they also observed a significant reduction in 90-day readmissions in the group with quieter rooms and improvements in patient satisfaction ratings on 'healthcare in general' and 'staff attitude'.

Vital signs may be collected too often and actually be detrimental to the patients' well-being and represent staff labour than can be diverted from low-risk patients to those at greater risk of physiological collapse. ${ }^{11}$ More frequent than usual (eg, every $4 \mathrm{~h}$ ) checks could be required to be written deliberately as orders rather than be the default setting for some patients. Where possible, continuous monitoring via new software-enabled devices that detect patient deterioration may also present a potential solution. Many hospital units, especially intensive care unit (ICUs), continue to use 24-h lighting, which should be considered a potential source of physiological stress. Hospitals should conduct noise studies, obtain more feedback from patients on the most disruptive sources of noise and aim to mitigate them. There may be even simpler interventions, such as encouraging use of personal or furnished noise-reduction headphones, eye masks, massage and music therapy, and placing art in hospital rooms to promote relaxation. Enabling patients to enjoy restful sleep through these simple interventions can dramatically improve the overall patient experience, and may reduce preventable harm caused by these hospital stressors.

\section{Implications for quality}

The hospital experience can be dehumanising, and increasing evidence points to the environment as central to quality. A telephone interview study of 380 discharged patients found that environment was the second-highest predictor of overall satisfaction with care, behind quality of clinical care. ${ }^{12}$ Many of the interventions described in this Viewpoint are easy to implement, low-risk and low-cost, while others will require extensive systems-level planning and careful implementation. Hospitals' bottom lines and patient satisfaction scores will ultimately benefit from overlooked details of care that improve the patient experience, especially as this experience becomes a publicly reported metric. Two questions on hospital environment, addressing noise and cleanliness, are now included in the Hospital Consumer Assessment of Healthcare Providers and Systems (HCAHPS) hospital survey and reported in public comparisons of hospitals published by the Centers for Medicare and Medicaid Services. The Joint Commission International has a standard that 'Patients at nutrition risk receive nutrition therapy' (Care of Patients 1.4), but sleep and rest are not yet addressed. ${ }^{13}$

The benefits of rest and nutrition may be underrepresented in the current medical literature. This is a field that may benefit from more interventional research to demonstrate the effectiveness of addressing a patient's basic needs. Nevertheless, hospitals can reduce these physiological stressors by designing more patient-centred hospital systems. As we seek to improve quality through patient-centredness, basic human needs are important in the context of complex medical care. We should view hospitals as healing environments rather than isolated clinical spaces and design patient care accordingly.

Contributors TX and MM drafted the manuscript. ECW provided critical review, comments, and the box 1 .

Competing interests None declared.

Provenance and peer review Not commissioned; internally peer reviewed.

Open Access This is an Open Access article distributed in accordance with the Creative Commons Attribution Non Commercial (CC BY-NC 4.0) license, which permits others to distribute, remix, adapt, build upon this work noncommercially, and license their derivative works on different terms, provided the original work is properly cited and the use is non-commercial. See: http://creativecommons.org/licenses/by$\mathrm{nc} / 4.0$ /

\section{REFERENCES}

1 Yosipovitch G, Tang M, Dawn AG, et al. Study of psychologic stress, sebum production and acne vulgaris in adolescents. Acta Derm Venereol 2007;87:135-9.

2 Makary MA, Segev DL, Pronovost PJ, et al. Frailty as a predictor of surgical outcomes in older patients. J Am Coll Surg 2010;210:901-8.

3 Detsky AS, Krumholz HM. Reducing the trauma of hospitalization. JAMA 2014;311:2169-70.

4 Powers J, Samaan K. Malnutrition in the ICU patient population. Cri Care Nurs Clin North Am 2014;26:227-42.

5 Martindale RG, McClave SA, Taylor B, et al. Perioperative nutrition: what is the current landscape? JPEN J Parenter Enteral Nutr 2013;37:5S-20S.

6 Apfelbaum JL, Caplan RA, Connis RT, et al. Practice guidelines for preoperative fasting and the use of pharmacologic agents to reduce the risk of pulmonary aspiration: application to healthy patients undergoing elective procedures: an updated report by the American Society of Anesthesiologists Committee on Standards and Practice Parameters. Anesthesiology 2011;114:495-511.

7 Lassen K, Soop M, Nygren J, et al. Consensus review of optimal perioperative care in colorectal surgery: Enhanced Recovery After Surgery (ERAS) Group recommendations. Arch Surg 2009;144:961-9.

8 Wick EC, Galante DJ, Hobson DB, et al. Organization culture changes result in improvement in patient-centered outcomes: 


\section{Viewpoint}

Implementation of an integrated recovery pathway for surgical patients. J Am Coll Surg 2015;221:669-77.

9 Johansson L, Berghom I, Waye KP, et al. The sound environment in an ICU patient room-a content analysis of sound levels and patient experiences. Intensive Crit Care Nurs 2012;28:269-79.

10 Hagerman I, Rasmanis G, Blomkvist V, et al. Influence of intensive coronary care acoustics on the quality of care and physiological state of patients. Int J Cardiol 2006;97:267-70.
11 Yoder JC, Yuen TC, Churpek MM, et al. A prospective study of nighttime vital sign monitoring frequency and risk of clinical deterioration. JAMA Intern Med 2013;173:1554-5.

12 Harris RH, McBride G, Ross C, et al. A place to heal: environmental sources of satisfaction among hospital patients. J Appl Soc Psychol 2002;32:1276-99.

13 Joint Commission. Joint Commission International accreditation standards for hospitals. 2014. http://www. jointcommissioninternational.org/assets/3/7/Hospital-5EStandards-Only-Mar2014.pdf 